\title{
Correlation of Apo B-48 and Apo B-100 with Oxidized LDL in Men with Central Obesity
}

\author{
Maria Diah Fibriani ${ }^{1,2}$, Andi Wijaya ${ }^{1,2}$, and Burhanuddin Bahar $^{3}$ \\ ${ }^{1}$ Post Graduate Program in Clinical Biochemistry, Hasanuddin University, Makassar \\ ${ }^{2}$ Prodia Clinical Laboratory, Jakarta \\ ${ }^{3}$ Faculty of Medicine, Hasanuddin University, Makassar
}

\section{Abstract}

$\mathrm{B}$ ACKGROUND: Obesity has a central role in the metabolic syndrome, which raises the risk for atherosclerotic cardiovascular disease (ASVCD). Apo B-48 and Apo B-100 are the necessary structural proteins required for the assembly and secretion of chylomicron and VLDL which have role in atherogenesis. The key initiating process in atherogenesis is the subendothelial retention of apolipoprotein B-containing lipoproteins. Oxidation of LDL is a hallmark of atherosclerosis development. The aim of this study was to asses the association between Apo B-48 and Apo B-100 with Oxidized-LDL as marker of atherosclerosis risk in central obesity. We hope that the result of this study can help to make a new strategy for the prevention and treatment of vascular disease.

RESULTS: There were 68 patients aged $39.6 \pm 7.3$ years, Apo B-48 concentration was $7.47 \pm 5.36 \mu \mathrm{g} / \mathrm{mL}$, Apo $\mathrm{B}-100$ was $117.26 \pm 25.74 \mathrm{mg} / \mathrm{dL}$, and ox-LDL was 137.05 $\pm 18.88 \mathrm{U} / \mathrm{L}$. This study showed a significant correlation between Apo B-100 and ox-LDL $(r=0.608, p<0.05)$ and correlation between Apo B-48 and ox-LDL ( $r=0.171$, $\mathrm{p}<0.05$ ). The levels of Apo B-100 were significantly different between obese with Mets and obese without Mets individuals $(\mathrm{p}<0.05)$.

CONCLUSIONS: This study suggested that Apo B-100 concentration increase in obese in Mets as compared with obese without Mets. Apo B-48 and Apo B-100 were correlated with Oxidized LDL, but correlation between Apo B-100 and ox-LDL more significant that Apo B-48 and $\mathrm{ox}-\mathrm{LDL}$.

KEYWORDS: Obesity, atherogenesis, Apo B-48, Apo B-100, ox-LDL.

\section{Introduction}

Obesity, now thought to affect 50 to 60 percent of a nation's population, is associated with insulin resistance and metabolic syndrome. Obesity contributes to hypertension, high serum cholesterol, low HDL-cholesterol and hyperglycaemia, and is independently associated with higher CVD risk (1). The accumulation of abdominal fat, which can be indirectly measured through waist circumference (WC), is an important coronary artery disease (CAD) risk factor (2).

Atherogenic dyslipidemia is one of the risk factors of Metabolic Syndrome, characterized by 3 lipoprotein abnormalities: elevated triglyceride, elevated small dense LDL particles, and low high-density-lipoprotein (HDL) cholesterol (3). Triglycerides in the vascular system are mainly transported by two distinct classes of triglyceride-rich lipoproteins (TRL), chylomicron and VLDL. The classes of TRL can be differentiated based on their apolipoproteins (apo), which serve to solubilize the hydrophobic lipid moiety (apo B100 and B48) (4). 
The apolipoproteins play minor roles as amphipathic proteins at the interface of the aqueous plasma and the lipid-soluble core of each lipoprotein particle, but they play major roles as regulators of the lipoprotein metabolism. However, apolipoprotein (apo) B48, which is synthesized in the intestine only, and apo B100, which is made in the liver, are the necessary structural proteins required for the assembly and secretion of chylomicrons and very lowdensity lipoproteins (VLDLs). Their secretion is regulated mainly by the avaibility of the core lipids (triglycerides and $\mathrm{CE}$ ) that they deliver into the circulation (5).

Apo B exists in two isoforms: apo B-100 and apo B-48. ApoB-100, a large glycoprotein with an approximate molecular mass of $550 \mathrm{kDa}$ is synthesized in the liver and is required for the assembly of very low density lipoproteins (VLDL). Apo B-100 has several functional and structural domains. It contains hydrophobic domains throughout its length that are believed to be involved in lipid binding. The carboxyl-terminal region of the protein has domains that bind to LDL receptors and to heparin $(6,7)$.

Apo B-48 is present in chylomicrons and chylomicrons remnants and plays an essential role in the intestinal absorption of dietary fats. Apo B-48 is synthesized in the small intestine and comprises the N-terminal $48 \%$ of apo $\mathrm{B}-100$ and is produced due to posttranscriptional apo B-100 mRNA editing at codon 2153 , which creates a stop codon in the intestine instead of a glutamine in the liver (7).

According to Tabas et al (2007) and Gustafsson and Boren (2004), the key initiating process in atherogenesis is the subendothelial retention of apolipoprotein $\mathrm{B}$-containing lipoproteins $(8,9)$. Retention of lipoproteins is the initiating step and leads to oxidation, inflammation, and endothelial dysfunction. The consequence of retention of atherogenic lipoproteins is not only a net accumulation of lipid, but also prolonged exposure to local oxidants and other nonoxidative enzymes in the vessel wall (9).

Within the intima lipoproteins can interact with the components of the arterial wall ECM, particularly proteoglycans. These macromolecules contain a core protein with attached carbohydrate chains consisting of negatively charged repeating disaccharide units called glycosaminoglycans, which form reversible ionic interactions with positively charged residues in atherogenic lipoproteins (10).

William and Tabas explain that positively charged residues in atherogenic lipoproteins are Apo B, the main protein moiety on LDL (11). Independent studies by Goldberg et al have shown that apolipoprotein B48 can interact with glycosaminoglycans (12).

Boren et al identified site B (residues 3359 to 3369) as the proteoglycan-binding site of apoB100 and showed that positively charged arginine and lysine residues of site B are critical for binding to CS/DS proteoglycans. Site B lies in the carboxyl-terminal half of Apo B100 and is absent in Apo B48. Flood et al in 2002 investigated the proteoglycan binding site of Apo B48 (B-Ib at residues 84 to 94), located in the amino terminus of apo $B$ and masked by the carboxyl terminus of Apo B100 in apo B100-containing LDL (13).

Oxidation of LDL is a hallmark of atherosclerosis development. Patients with manifest $\mathrm{CAD}$ have elevated plasma concentration of circulating ox-LDL that is associated with the severity of symptoms and degree of CAD (1).

LDL modified chemically is readily internalized by macrophages through a so-called "scavenger receptor" pathway. It is now clear that one mechanism whereby cells in vitro render LDL a substrate for the scavenger receptor pathway is via oxidation of LDL lipids and the resulting modification of apolipoprotein B-100. These observations form the basis for the oxidative modification hypothesis of atherosclerosis in which LDL traverses the subendothelial space of lesion-prone arterial sites. During this process, LDL lipids are subject to oxidation and, as a consequence, apolipoprotein B-100 lysine groups are modified so that the net negative charge of the lipoprotein particle increases. This modification of apolipoprotein B-100 renders LDL susceptible to macrophage uptake via a number of scavenger receptor pathways producing cholesterol ester-laden foam cells. It is this accumulation of foam cells that forms the nidus of a developing atherosclerotic lesion (14).

The aim of this study was to asses the association between Apo B-48 and Apo B-100 with Oxidized-LDL as marker of atherosclerosis risk in central obesity. We hope that the result of this study can help to make a new strategy for the prevention and treatment of vascular disease.

\section{Patients and Methods}

The study applied the cross sectional method, in which the study subjects were 68 adult males, aged $25-55$ years, with central obesity (waist circumference $>90 \mathrm{~cm}$ ). The subjects were divided into Mets subjects and non-Mets subjects (based on IDF 2005 criteria).

\section{ASSAY OF BIOCHEMICAL MARKERS}

The measurement of Apo B-100 was done by homogenous method Daichii reagent kit, Japan. The measurement of Apo B-48 was done using ELISA method Biovendor reagent kit, Japan. Meanwhile ox-LDL was measured by 
using sandwich/capture ELISA method Mercodia reagent, Swedia. All assays were performed according to the manufacturers' instructions.

For each run of Apo B-48, Apo B-100, and ox-LDL, controls were included in the assays, and all results were within acceptable ranges.

\section{STATISTICAL ANALYSIS}

Statistical analysis was performed with the SPSS for Windows version 13,0 software. Univariate, bivariate, and multivariate analyses were calculated. The results were presented in narration as well as in tables and graphics. For statistical differences tests we used $5 \%$ significance level

\section{Results}

The study subjects consisted of 38 Mets $(55,88 \%)$ and 30 non-Mets $(44,12 \%)$ based on IDF 2005 criteria.

Table 1. Basic characteristics of the study subjects

\begin{tabular}{lcccc}
\hline \multicolumn{1}{c}{ Variables } & N & Min & Max & Mean \pm SD \\
\hline Age (years) & 68 & 25 & 55 & $39,60 \pm 7,27$ \\
Height $(\mathrm{cm})$ & 68 & 157 & 182 & $168,12 \pm 5,56$ \\
Body weight $(\mathrm{kg})$ & 68 & 62 & 112 & $81,44 \pm 10,78$ \\
BMI $\left(\mathrm{kg} / \mathrm{m}^{2}\right)$ & 68 & 23,05 & 41,64 & $28,79 \pm 3,35$ \\
Waist circumference $(\mathrm{cm})$ & 68 & 90 & 120 & $99,81 \pm 7,48$ \\
Blood Pressure & & & & \\
Diastolic $(\mathrm{mmHg})$ & 68 & 90 & 180 & $124,71 \pm 18,16$ \\
Systolic $(\mathrm{mmHg})$ & 68 & 70 & 120 & $84,63 \pm 11,70$ \\
Fasting glucose $(\mathrm{mg} / \mathrm{dL})$ & 68 & 16 & 316 & $105,40 \pm 41,28$ \\
Total Cholest $(\mathrm{mg} / \mathrm{dL})$ & 68 & 43 & 334 & $218,07 \pm 41,72$ \\
Triglyceride $(\mathrm{mg} / \mathrm{dL})$ & 68 & 62 & 408 & $193,32 \pm 81,11$ \\
LDL Cholest $(\mathrm{mg} / \mathrm{dL})$ & 68 & 26 & 264 & $140,51 \pm 34,89$ \\
HDL Cholest $(\mathrm{mg} / \mathrm{dL})$ & 68 & 71 & 62 & $42,96 \pm 7,24$ \\
Apo B-10mm $(\mathrm{mg} / \mathrm{dL})$ & 68 & 1,00 & 201 & $117,26 \pm 25,74$ \\
Apo B-48 $(\mu \mathrm{gg} / \mathrm{mL})$ & 68 & 74,73 & 26,12 & $7,47 \pm 5,36$ \\
Ox-LDL $(\mathrm{U} / \mathrm{l})$ & 68 & & 158,31 & $137,05 \pm 18,88$ \\
\hline
\end{tabular}

BMI = Body Mass Index; HDL = High Density Lipoprotein; LDL = Low Density Lipoprotein; Apo = Apolipoprotein; ox-LDL = oxidized Low Density Lipoprotein, $\mathrm{SD}=$ Standard Deviation.

Table 2. Correlation between Apo B-48, Apo B-100 and ox-LDL

\begin{tabular}{ccc}
\hline Variable & rs & p \\
\hline Apo B-100 - Ox-LDL & 0,608 & $0,000^{\star \star}$ \\
Apo B-48 - Ox-LDL & 0,171 & $0,013^{\star}$ \\
\hline
\end{tabular}

rs = correlation coefficient Spearman

** = significant $(p<0.01)$

* $=$ significant $(p<0.05)$ 


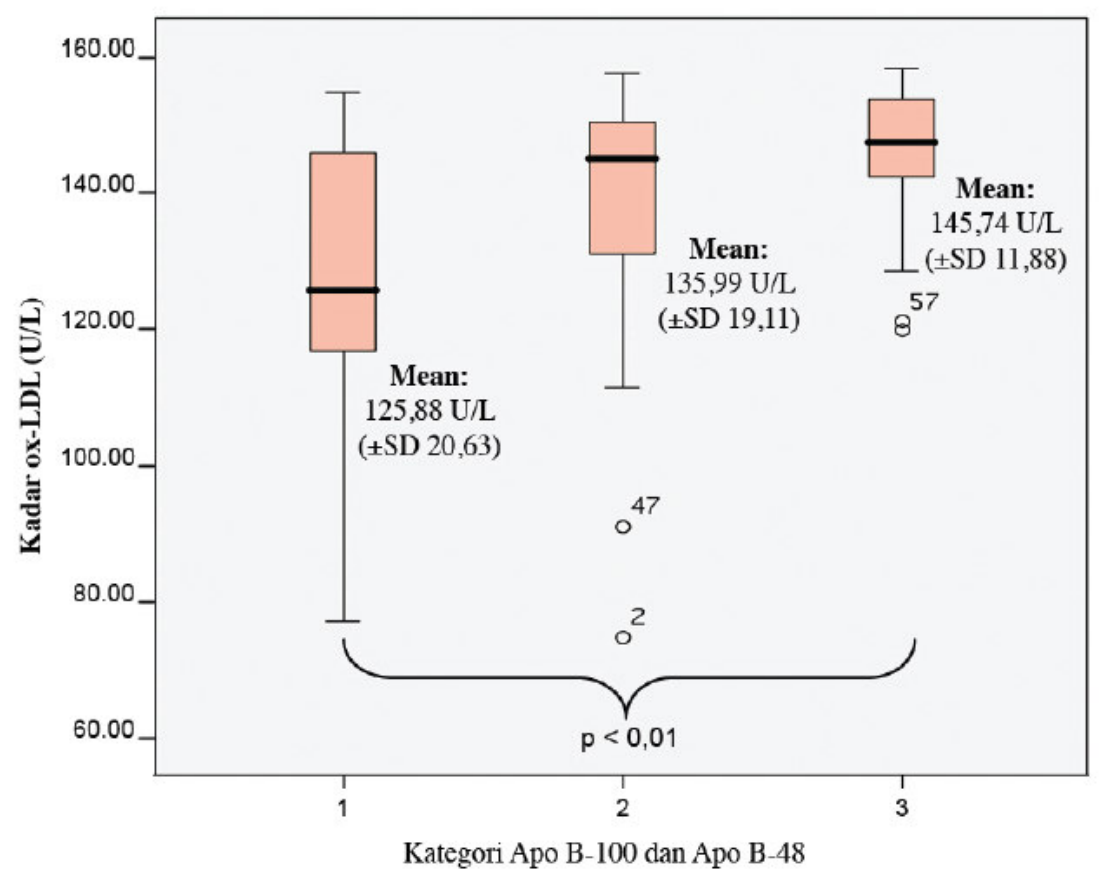

Category 1 = Apo B-100 and Apo B-48 < Median, 2 = Apo B-100 < Median and Apo B-48 > Median or Apo B-100 > Median and Apo B-48 < Median, 3 = Apo B-100 and Apo B-48 > Median, SD = Standart Deviation

Figure 1. The correlation of the combination of Apo B-48-Apo B-100 and Ox-LDL

Table 3. Differences tests of Mets and Non Mets groups

\begin{tabular}{lccc}
\hline \multicolumn{1}{c}{$\begin{array}{c}\text { Characteristics } \\
\text { Mean } \pm \text { SD }\end{array}$} & Obese with Mets & Obese without Mets & p \\
\hline Age (years) & $39,18 \pm 6,84$ & $40,13 \pm 7,88$ & 0,603 \\
Height $(\mathrm{cm})$ & $168,45 \pm 6,14$ & $167,70 \pm 4,81$ & 0,575 \\
Body weight $(\mathrm{kg})$ & $83,34 \pm 12,18$ & $79,03 \pm 8,25$ & 0,088 \\
BMI $\left(\mathrm{kg} / \mathrm{m}^{2}\right)$ & $29,33 \pm 3,71$ & $28,11 \pm 2,73$ & 0,123 \\
Waist circumference $(\mathrm{cm})$ & $101,16 \pm 8,65$ & $98,10 \pm 5,33$ & 0,078 \\
Blood Pressure & & & \\
Diastolic $(\mathrm{mmHg})$ & $131,05 \pm 19,84$ & $116,67 \pm 11,84$ & $<0.001$ \\
Systolic $(\mathrm{mmHg})$ & $88,82 \pm 19,84$ & $79,33 \pm 11,84$ & $<0.001$ \\
Fasting glucose $(\mathrm{mg} / \mathrm{dL})$ & $117,66 \pm 51,94$ & $89,87 \pm 7,00$ & $0,002^{*}$ \\
Total Cholest $(\mathrm{mg} / \mathrm{dL})$ & $224,21 \pm 46,25$ & $210,30 \pm 34,35$ & 0,160 \\
Triglyceride $(\mathrm{mg} / \mathrm{dL})$ & $234,66 \pm 74,67$ & $140,97 \pm 54,81$ & $<0.001$ \\
LDL Cholest $(\mathrm{mg} / \mathrm{dL})$ & $140,66 \pm 40,04$ & $140,33 \pm 27,68$ & 0,969 \\
HDL Cholest $(\mathrm{mg} / \mathrm{dL})$ & $41,08 \pm 6,39$ & $45,33 \pm 7,64$ & $0,018^{*}$ \\
Apo B-10mm $(\mathrm{mg} / \mathrm{dL})$ & $122,92 \pm 28,29$ & $110,10 \pm 20,36$ & $0,034^{*}$ \\
Apo B-48 $(\mu \mathrm{g} / \mathrm{mL})$ & $8,30 \pm 5,71$ & $6,42 \pm 4,76$ & 0,143 \\
Ox-LDL $(\mathrm{U} / \mathrm{l})$ & $139,21 \pm 17,01$ & $134,32 \pm 20,99$ & 0,306 \\
\hline
\end{tabular}

${ }^{*} p<0,05$ : significant. 


\section{Discussion}

In this study, Apo B-100, Apo B-48 concentration similar with previous studies $(15,16)$, but ox-LDL concentration higher than ox-LDL concentration in Weinbrenner et.al study (2.) Based on the correlation coefficient and probability, the correlation between Apo B-100 and oxLDL was more significant than that of Apo B-48 and oxLDL.

The elevated concentration of free fatty acid that accumulates in the liver in central obesity causes increased production of VLDL and formation of VLDL rich in triglyceride. The high concentration of VLDL in circulation increases the exchange of triglyceride-VLDL for esterification of cholesterol in HDL and LDL particles. The triglyceride transferred to LDL and HDL makes this particle change into a good substrate for hepatic lipase. The increased activity of hepatic lipase results in the formation of small dense LDL and the decrease of anti atherogenic HDL $(17,18)$. VLDL, LDL, and small dense LDL containing Apo B-100, hence in subjects with central obesity there is an increase of Apo B-100 concentration. Because Apo B-100 is positively charged, it becomes easy to bind to subendothelial proteoglycan that is negatively charged. The binding of Apo B-100 to proteoglycan results in retention of lipoprotein in the subendothelial. The retention of lipoproteins is the initiating step to oxidation, inflammation, and endothelial dysfunction. The consequence of retention of atherogenic lipoproteins is not only a net accumulation of lipid, but also prolonged exposure to local oxidants and other non-oxidative enzymes in the vessel wall $(9,11)$. The oxidative changes of LDL into oxidized LDL are the biological key processes which are the start and accelerator of atherosclerosis process leading to the formation of fatty streak. The binding oxLDL to macrophages is an important stage, in which oxLDL accelerates cholesterol accumulation and changes macrophages to become foam cells (19).

The increase of triglyceride in central obesity can increase the Triglyceride Rich Lipoprotein including chylomicron in the intestine. Chylomicron is subsequently hydrolyzed by Lipoprotein Lipase in plasma to become remnant chylomicron. Chylomicron and remnant chylomicron contain Apo B-48 which is positively charged. Similar to LDL, apolipoprotein B-48-containing chylomicron remnants may also bind to proteoglycans of the apolipoprotein and thus retained within the arterial wall (20)
Based on these data, we wanted to assess the correlation of the combination of Apo B-48-Apo B-100 and Ox-LDL. Figure 1 shows that the higher the concentration of both parameters, the higher the concentration of Ox-LDL. The role of Apo B-48 and Apo B-100 in atherosclerosis is mostly related to their positively charges, and as such they easily bind and change into proteoglycan in the intima, which plays key role in the start of atherosclerosis $(8,9$, 11).

Table 3 shows results of the differences tests on the Mets group and Non Mets group. There were significant differences of Diastolic, Systolic, Fasting glucose, Triglyceride, HDL, Apo B-100, respectively. Although there was no significant difference, the mean of Apo B-4 8 and Ox-LDL was higher in Mets group than that in the non-Mets group.

The parameters of Diastolic, Systolic, Fasting glucose, Triglyceride, and HDL cholesterol are the components of Metabolic Syndrome, and therefore there is a significant difference of Mets group and Non Mets group.

The differences of Apo B-100 of Mets group and Non Mets group were similar to the result of the study by Sniderman (21), where subjects with high Apo B-100 were more obese with obvious manifestations of the metabolic syndrome as compared with those who had high LDL cholesterol; Apo B-100 was more related with central obesity, insulin resistance, and inflammation as compared with non-HDL cholesterol, and Apo B-100 was a better parameter to identify individuals with or without metabolic syndrome with the increased risk for cardiovascular event (22); and Metabolic Syndrome was related to higher concentration of Apo B-100 (odds ratio 2.97) (23). In this study, the Odds Ratio of Apo B-100 was 1.591 ( $\mathrm{p}<0.001$ ).

Apo B-100 was more closely associated than Non HDL Cholesterol with central adiposity, insulin resistance, thrombosis, and inflammation, and apo B- 100 was a better candidate for risk parameter than Non HDL Cholesterol for identifying a subgroup of individuals with or without MetS with elevated cardiovascular risks (22).

One of the risk factors of Metabolic Syndrome is the Atherogenic Dyslipidemia, comprising increase of triglyceride, decrease of HDL-cholesterol, and the presence of small dense LDL. One of the lipoprotein-rich triglycerides is VLDL which contains Apo B-100, small dense LDL as well as Apo B-100, so the concentration of Apo B-100 in Mets group is higher than in Non-Mets group.

Although there is no significant difference, the mean of Apo B-4 8 and Ox-LDL is higher in Mets group than in non Mets group. The increase of triglyceride in 
metabolic syndrome causes the increase of Apo B-48 containing chylomicron and remnant chylomicron, thus the concentration of Apo B-48 in Mets group is higher than in Non-Mets group. The increase of Apo B-48 and Apo B-100 in metabolic syndrome relates with atherogenic lipoprotein retention, hence an increased atherosclerosis risk (marked by ox-LDL).

\section{Conclusions}

1. Apo B-100 has more significant correlation with increased atherosclerosis risks.

2. Apo B-100 in Mets group has significant difference with non Mets group.

\section{Acknowledgements:}

Thanks are due to the Prodia Foundation for Research and Training for the invaluable supports given throughout this study.

\section{References:}

1. International Diabetes Federation. Consensus on the Metabolic Syndrome: Definition and Treatment. Berlin. 2005

2. Weinbrenner, T., Schroder, H., Escurriol, V., Fito, M., Elosua, R., Vila, J., Marrugat, J., Covas, M.I. Circulating OxLDL is Associated with Increased Waist Circumference Independent of Body Mass Index in Men and Women. Am J Clin Nutr 2006; 83:30-5

3. Rizzo M, Berneis K. Small, dense low-density-lipoproteins and the metabolic syndrome. Diabetes Metab Res Rev 2007; 23:14-20

4. Heeren, J., Beisiegel, U. Intracellular Metabolism of TriglycerideRich Lipoproteins. Curr Opin Lipidol 2001; 12: 255-260

5. Ginsberg, H.N. New Perspectives on Atherogenesis, Role of Abnormal Triglyceride-Rich Lipoprotein Metabolism. Circulation 2002; 106: 2137-2142

6. Young, S.G. Recent Progress in Understanding Apolipoprotein B. Circulation 1990; 82:1574-15942

7. Whitfield, A.J., Barret, H.R., Bockxmeer, M.V., Burnett, J.R. Lipid Disorders and Mutation in the ApoB Gene. Clinchem 2004; $50: 10$

8. Tabas, I., Williams, K.J., Boren, J. Subendothelial Lipoprotein Retention as the Initiating Process in Atherosclerosis. Circulation 2007; 116:1832-1844

9. Gustafsson, M. and Boren, J. Mechanism of Lipoprotein Retention by the Extracellular Matrix, Curr Opin Lipidol 2004; 15:505-514
10. Camejo, G., Hurt-Camejo, E., Wiklund, O., Bondjers, G. Association of Apo B Lipoproteins with Arterial Proteoglycans : Pathological Significance and Molecular Basis. Atherosclerosis 1998; 139:205-222

11. Williams, K.J., Tabas, I. The Response-to-Retention Hypothesis of Early Atherogenesis. Arterioscler Thromb Vasc Bio 1995; 15:551-561

12. Goldberg, I.J., Wagner, W.D., Pang, L., Paka, L., Curtis, L.K., DeLozier, J.A., Shelness, G.S. The NH2-Terminal Region of Apolipoprotein B is Sufficient for Lipoprotein Association with Glycosaminoglycans. J. Biol Chem 1998; 273:3535535361

13. Boren, J., Olin, K., Lee, I., Chait, A., Wight, T.N., Innerarity, T.L. Identification of the Principal Proteoglycan-Binding Site in LDL. A Single-Point Mutation in Apo-B100 Severely Affects Proteoglycan Interaction without Affecting LDL Receptor Binding. J Clin Invest 1998; 101:2658-2664

14. Stocker, R. and Keaney, J.F. Role of Oxidative Modifications in Atherosclerosis. Physiol Rev 2004; 84:1381-1478

15. Onat A, Can G, Hergenc G, Yazici M, Karabulut A, Albayrak S. Serum Apolipoprotein B Predicts Dyslipidemia, Metabolic Syndrome and, in Women, Hypertension and Diabetes, Independent of Markers of Central Obesity and Inflammation. International Journal of Obesity 2007; 31 : 1119-1125

16. Sakai N, Uchida $\mathrm{Y}$, Ohashi $\mathrm{K}$, et.al. Measurement of Fasting Serum apoB-48 Levels in Normolipidemic and Hyperlipidemic Subjects by ELISA. Journal of Lipid Research 2003; 44:1256-1262

17. Grundy, S.M., Abate, N. Secondary Heart Disease : Systemic Diseases and the Heart Obesity 2003; 8(2):1-6

18. Carr, M.C., Brunzell, J.D. Abdominal Obesity and Dyslipidemia in the Metabolic Syndrome : Importance of Type 2 Diabetes and Familial Combined Hyperlipidemia in Coronary Artery Disease Risk. J Clin. Endocrinol Metab 2004; 89(6):26012607

19. Holvoet, P., Vanhaecke, J., Janssens, S., Van de Werf, F., Collen, D. Oxidized LDL and Malondialdehyde-Modified LDL in Patients With Acute Coronary Syndromes and Stable Coronary Artery Disease. Circulation 1998; 98:1487-1494

20. Proctor, S.D., Vine, D.F., Mamo, J.C.L. Arterial Retention of Apolipoprotein B48 and B100-containing Lipoproteins in Atherogenesis. Curr Opin Lipidol. 2002; 13: 461-470

21. Sniderman AD, St-Pierre AC, Cantin B, Dagenais GR, Despres JP, Lamarche B. Concordance/discordance between Plasma Apolipoprotein B Levels and the Cholesterol Indexes of Atherosclerotic Risk. Am J Cardiol 2003; 91: 1173-1177

22. Sattar, N., Williams, K., Sniderman, A.D., D' Agostino, R. Jr., Haffner, S.M. Comparison of Apo B and non-HDL Cholesterol with other Cardiovascular Risk Factors in Patients with the Metabolic Syndrome in the Insulin Resistance Atherosclerosis Study (IRAS). Circulation 2004; 110: 2687-2693

23. Muntner $\mathrm{P}, \mathrm{HeJ}$, Chen J, Fonseca V, Whelton PK. Prevalence of Non-traditional Disease Risk Factors among Persons with Impaired Fasting Glucose, Impaired Glucose Tolerance, Diabetes, and the Metabolic Syndrome: Analysis of the Third National Health and Nutrition Examination Survey (NHANES III]. Ann Epidemiol 2004; 14: 686-695 